\title{
Tourette Syndrome
}

National Institute of Neurological Disorders and Stroke (NINDS)

\section{Source}

National Institute of Neurological Disorders and Stroke (NINDS). Tourette Syndrome

Information Page.

Tourette syndrome (TS) is a neurological disorder characterized by repetitive,

stereotyped, involuntary movements and vocalizations called tics. The first symptoms of

TS are almost always noticed in childhood. Some of the more common tics include eye blinking and other vision irregularities, facial grimacing, shoulder shrugging, and head or shoulder jerking. Perhaps the most dramatic and disabling tics are those that result in self-harm such as punching oneself in the face, or vocal tics including coprolalia (uttering swear words) or echolalia (repeating the words or phrases of others). Many with TS experience additional neurobehavioral problems including inattention, hyperactivity and impulsivity, and obsessive-compulsive symptoms such as intrusive thoughts/worries and repetitive behaviors. 